\title{
Corrigendum: A Synthetic Small Molecule F240B Decreases NLRP3 Inflammasome Activation by Autophagy Induction
}

\begin{abstract}
Chun-Hsien Wu ${ }^{1,2}$, Chin Heng Gan ${ }^{3}$, Lan-Hui $L^{4,5}{ }^{4}$, Jen-Che Chang ${ }^{6}$, Shin-Tai Chen ${ }^{6}$, Mridula P. Menon ${ }^{6}$, Shu-Meng Cheng ${ }^{1}$, Shih-Ping Yang ${ }^{1}$, Chen-Lung $\mathrm{Ho}^{7}$, Oleg V. Chernikov ${ }^{8}$, Chi-Hung Lin ${ }^{2,9}$, Yulin Lam ${ }^{3^{*}}$ and Kuo-Feng Hua ${ }^{5,6,10^{*}}$

1 Division of Cardiology, Department of Internal Medicine, Tri-Service General Hospital, National Defense Medical Center, Taipei, Taiwan, ${ }^{2}$ Institute of Microbiology and Immunology, National Yang-Ming University, Taipei, Taiwan, ${ }^{3}$ Department of Chemistry, National University of Singapore, Singapore, Singapore, ${ }^{4}$ Department of Laboratory Medicine, Linsen, Chinese Medicine and Kunming Branch, Taipei City Hospital, Taipei, Taiwan, ${ }^{5}$ Department of Pathology, Tri-Service General Hospital, National Defense Medical Center, Taipei, Taiwan, ${ }^{6}$ Department of Biotechnology and Animal Science, National llan University, Ilan, Taiwan, ${ }^{7}$ Division of Wood Cellulose, Taiwan Forestry Research Institute, Taipei, Taiwan, ${ }^{8}$ G.B. Elyakov Pacific Institute of Bioorganic Chemistry FEB RAS, Vladivostok, Russia, ${ }^{9}$ Department of Biological Science \& Technology, National Chiao Tung University, Hsinchu, Taiwan, 10 Department of Medical Research, China Medical University Hospital, China Medical University, Taichung, Taiwan
\end{abstract}

Keywords: NLRP3 inflammasome, conjugated polyenes, autophagy, mitochondria, peritonitis

\section{OPEN ACCESS}

Edited and reviewed by: Massimo Collino,

University of Turin, Italy

*Correspondence: Kuo-Feng Hua kuofenghua@gmail.com Yulin Lam

chmlamy|@nus.edu.sg

Specialty section: This article was submitted to Inflammation,

a section of the journal

Frontiers in Immunology

Received: 09 July 2021 Accepted: 20 October 2021 Published: 15 December 2021

Citation:

Wu C-H, Gan CH, Li L-H, Chang J-C, Chen S-T, Menon MP, Cheng S-M,

Yang S-P, Ho C-L, Chernikov OV, Lin C-H, Lam Y and Hua K-F (2021) Corrigendum: A Synthetic Small Molecule F240B Decreases NLRP3

Inflammasome Activation by Autophagy Induction.

Front. Immunol. 12:738591. doi: 10.3389/fimmu.2021.738591

\section{A Corrigendum on:}

A Synthetic Small Molecule F240B Decreases NLRP3 Inflammasome Activation by Autophagy Induction by Wu C-H, Gan CH, Li L-H, Chang J-C, Chen S-T, Menon MP, Cheng S-M, Yang S-P, Ho C-L, Chernikov OV, Lin C-H, Lam Y and Hua K-F (2020). Front. Immunol. 11:607564. doi: $10.3389 /$ fimmu.2020.607564

In the original article, there was a mistake in Figures $\mathbf{5 E}$ and $\mathbf{8 E}$ as published. We found a mismatch between the Western blot images ( $\mathrm{p}-\mathrm{JNK} 1 / 2)$ in comparison to the labeling in Figures $\mathbf{5 E}$. Regarding the effect of F240B on LPS-induced phosphorylation of ERK1/2, p38 and JNK1/2, we tested two time-course studies: (A) condition was LPS treatment for 0, 10, 20, $30 \mathrm{~min}$ (total eight groups), (A) condition was LPS treatment for $0,10,20,30,60 \mathrm{~min}$ (total ten groups). In the Figures 5E of the original manuscript, we used (A) condition for ERK1/2, p38; however, we used (B) condition for JNK. In addition, in Figures 8E of the original manuscript, the Western blot image of input NLRP3 included a non-specific band (far left band), making it looks like a mismatch between input NLRP3 and input PKR. The corrected Figures 5E and 8E appear below.

The authors apologize for this error and state that this does not change the scientific conclusions of the article in any way. The original article has been updated.

Publisher's Note: All claims expressed in this article are solely those of the authors and do not necessarily represent those of their affiliated organizations, or those of the publisher, the editors and the reviewers. Any product that may be evaluated in this article, or claim that may be made by its manufacturer, is not guaranteed or endorsed by the publisher.

Copyright (C) 2021 Wu, Gan, Li, Chang, Chen, Menon, Cheng, Yang, Ho, Chernikov, Lin, Lam and Hua. This is an open-access article distributed under the terms of the Creative Commons Attribution License (CC BY). The use, distribution or reproduction in other forums is permitted, provided the original author(s) and the copyright owner(s) are credited and that the original publication in this journal is cited, in accordance with accepted academic practice. No use, distribution or reproduction is permitted which does not comply with these terms. 
A

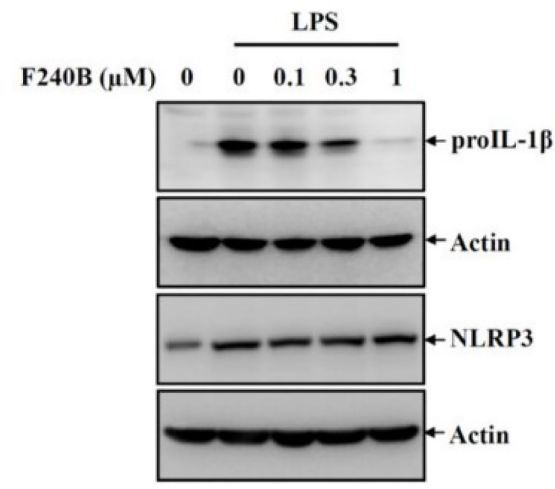

$\mathbf{E}$

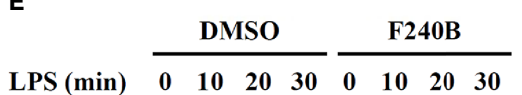

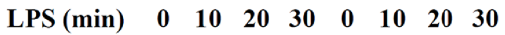

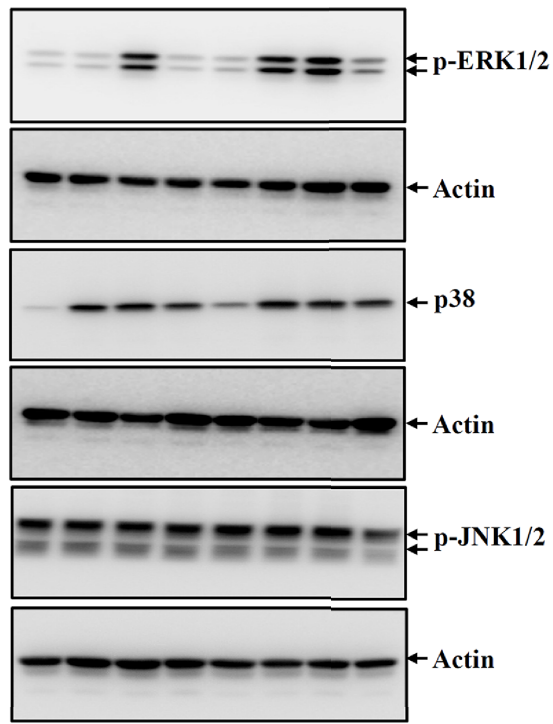

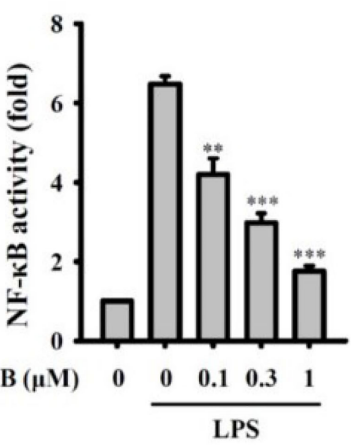

C

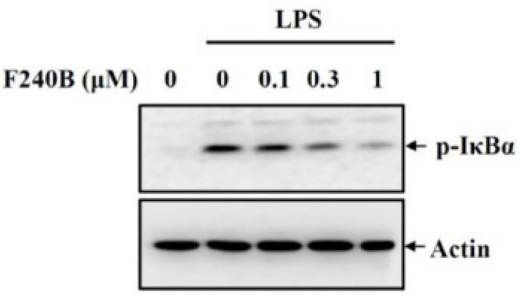

D

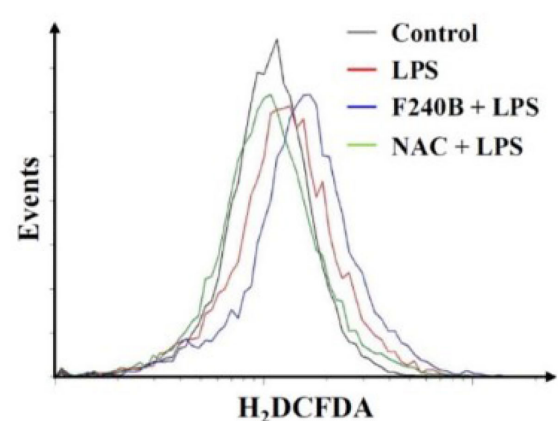

$\mathbf{F}$

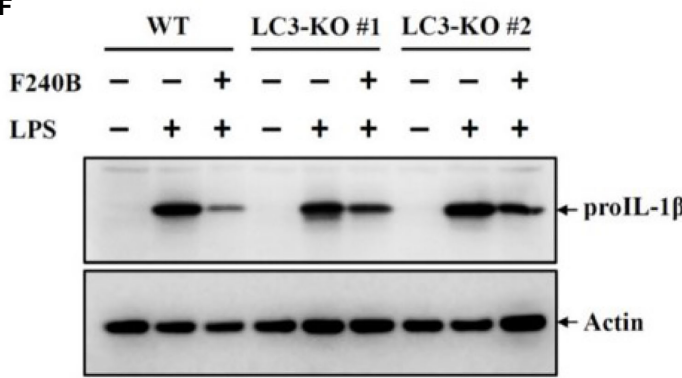

FIGURE 5 | Activation of autophagy by F240B inhibits NF-kB activation and prolL-1b expression. (A) J774A.1 macrophages were incubated with F240B for $0.5 \mathrm{~h}$ followed by incubated with $1 \mu \mathrm{g} / \mathrm{ml}$ LPS for $6 \mathrm{~h}$. The levels of prolL-1b and NLRP3 in the cell lysates were measured by Western blotting. (B) J-Blue cells were incubated with F24OB for $0.5 \mathrm{~h}$ followed by incubated with $1 \mu \mathrm{g} / \mathrm{ml}$ LPS for $24 \mathrm{~h}$. The NF-kB transcriptional activity was measured by NF-kB reporter assay. (C, D) J774A.1 macrophages were incubated with F240B (1 $\mu \mathrm{M}$ for ROS assay) for $0.5 \mathrm{~h}$ followed by incubated with $1 \mu \mathrm{g} / \mathrm{ml}$ LPS for $10 \mathrm{~min}$. The phosphorylation levels of $\mathrm{kBB}$ in the cell lysates were measured by Western blotting (C), and the intracellular ROS production was analysed by H2DCFDA staining (D). (E) J774A.1 macrophages were incubated with $1 \mu \mathrm{M} \mathrm{F} 240 \mathrm{~B}$ for $0.5 \mathrm{~h}$ followed by incubated with $1 \mu \mathrm{g} / \mathrm{ml}$ LPS for 10-30 min. The phosphorylation levels of ERK1/2, JNK1/2 and p38 in the cell lysates were measured by Western blotting. (F) Will-type or LC3-knockout J774A.1 macrophages were incubated with $1 \mu \mathrm{M}$ F240B for $0.5 \mathrm{~h}$ followed by incubated with $1 \mu \mathrm{g} / \mathrm{ml}$ LPS for $6 \mathrm{~h}$. The levels of prolL-1b in the cell lysates were measured by Western blotting. ${ }^{\star \star}$ and ${ }^{* \star \star}$ indicate a significant difference at the level of $p<0.01$ and $p<0.001$, respectively compared to LPS. 
A
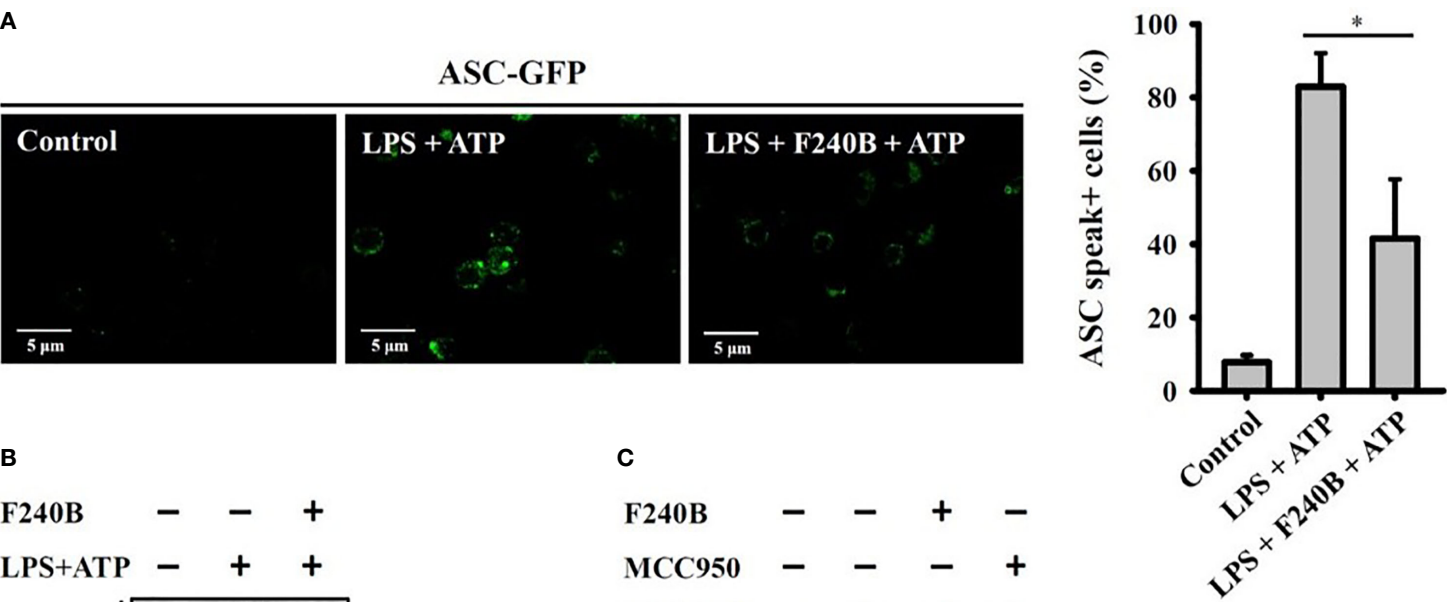

B

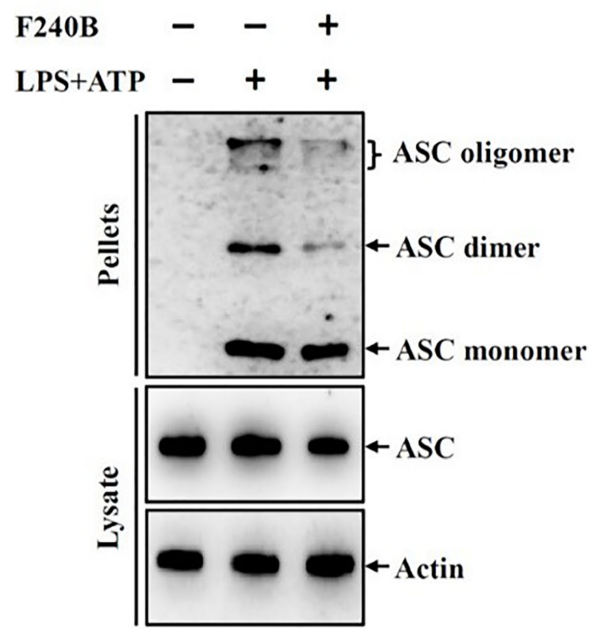

C

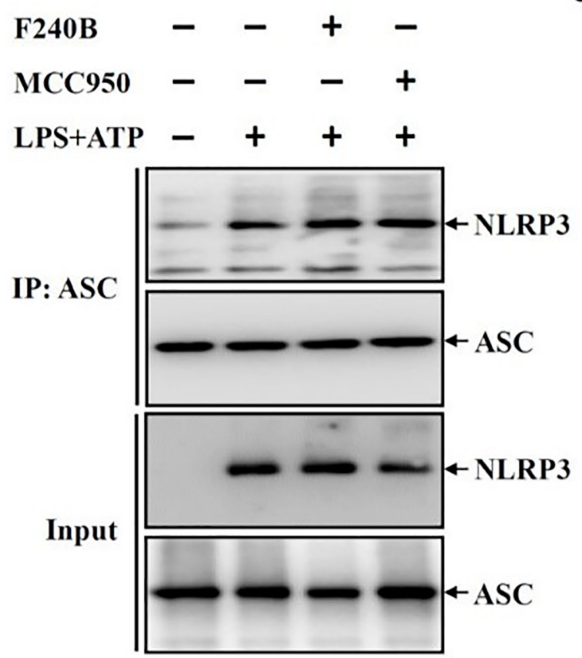

D

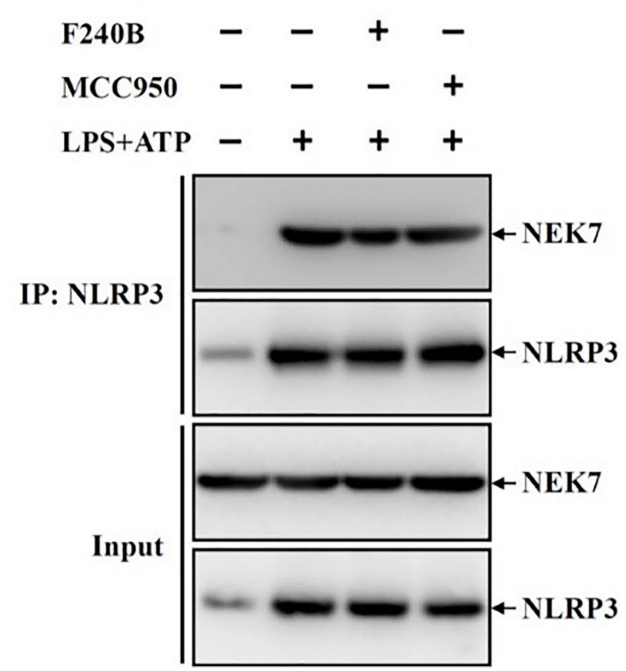

E

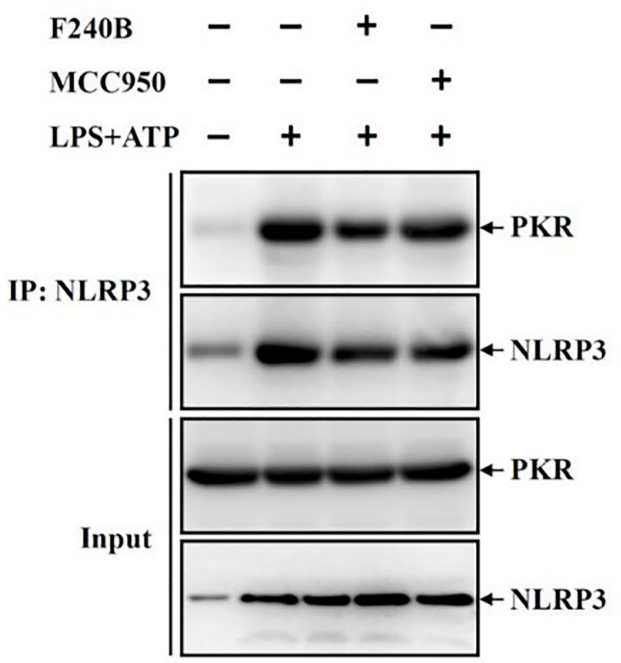

FIGURE 8 | F240B inhibits ASC oligomerization. (A) ASC-GFP expressed J774A.1 macrophages or (B) J774A.1 macrophages were incubated with $1 \mu \mathrm{g} / \mathrm{ml}$ LPS for $5 \mathrm{~h}$ followed by incubated with $1 \mu \mathrm{M} \mathrm{F} 240 \mathrm{~B}$ for $3 \mathrm{~h}$. Cells then incubated with $5 \mathrm{mM}$ ATP for $0.5 \mathrm{~h}$. The ASC speck formation was analyzed by fluorescent microscope (A), or the cell lysates were crosslinked by disuccinimidyl suberate and ASC oligomerization was analyzed by Western blotting (B). (C-E) J774A.1 macrophages were incubated with $1 \mu \mathrm{g} / \mathrm{ml}$ LPS for $5 \mathrm{~h}$ followed by incubated with $1 \mu \mathrm{M} \mathrm{F240B}$ or $0.1 \mu \mathrm{M}$ MCC950 for $3 \mathrm{~h}$. Cells then incubated with $5 \mathrm{mM}$ ATP for $0.5 \mathrm{~h}$. The interaction between NLRP3 with ASC (C), NEK7 (D) or PKR (E) were analyzed by immunoprecipitation and Western blotting assay. The percentage of ASC speck positive cells are expressed as the mean \pm SD of three separate experiments. * indicates a significant difference at the level of $p<0.05$. 